\title{
Effects of abnormal activation on the time course of the left ventricular pressure pulse in dilated cardiomyopathy
}

\author{
Han B Xiao, Stephen J D Brecker, Derek G Gibson
}

\begin{abstract}
Objective-To investigate the effects of QRS duration on characteristics of the left ventricular pressure pulse derived from the time course of functional mitral regurgitation by continuous wave Doppler.

Design-Retrospective and prospective study of 50 patients with dilated cardiomyopathy, by electrocardiography, echocardiography, and Doppler cardiography.

Setting-Tertiary cardiac referral centre.
\end{abstract}

Patients-50 patients (mean age (SD) 58 (16)) with dilated cardiomyopathy, all with functional mitral regurgitation.

Results-The values of QRS duration ranged widely, from 70 to 190 ms with a mean value of $110 \mathrm{~ms}$, and were unimodally distributed. The overall duration of mitral regurgitation correlated positively with QRS time $(r=0.65)$ over the entire range of values. When the duration of mitral regurgitation was divided into contraction, aortic ejection, and relaxation times, increased $Q R S$ duration prolonged contraction $(r=0.51)$ and relaxation $(r=0.52)$ times. Aortic ejection time was affected by $R R$ interval $(r=0 \cdot 74)$. Duration of QRS correlated negatively with peak rate of rise in left ventricular pressure $(+\mathrm{dP} / \mathrm{dt})$ $(r=-0.48)$, and positively with the time intervals from $Q$ to peak pressure $(r=0.49)$ and to peak $+\mathrm{dP} / \mathrm{dt}(r=0.72)$, and also with those from the start of mitral regurgitation to peak pressure $(r=0.49)$ and to peak $+\mathrm{dP} / \mathrm{dt}(r=0.76)$. Duration of QRS did not directly affect the peak rate of left ventricular pressure fall ( $-\mathrm{dP} / \mathrm{dt})$, or the isovolumic relaxation period.

Conclusions-Values of QRS duration are unimodally distributed in patients with dilated cardiomyopathy, without evidence of a discrete group of patients with left bundle branch block. Prolonged QRS duration reduces peak $+\mathrm{dP} / \mathrm{dt}$, prolongs overall duration of the pressure pulse, the time to peak + dP/dt, and relaxation time. Duration of QRS must therefore be taken into account in assessing standard measurements of myocardial function in patients with dilated cardiomyopathy.

(Br Heart J 1992;68:403-7)
In 1926, Wiggers ${ }^{1}$ questioned whether changes in ventricular conduction were important in determining the dynamics of ventricular contraction. He concluded that intraventricular pressure curves could only be regarded as reflecting the contraction of the individual muscle units of the ventricle as long as excitation passed over natural pathways from a supraventricular focus. Since then, the question has been investigated little, particularly as it applies clinically, and yet it remains an important one. In human left ventricular disease, major disturbances of ventricular activation may occur, yet changes in the pressure pulse are often used to assess myocardial function. ${ }^{23}$ Peak rate of the rise of pressure standardised to instantaneous pressure has been suggested as an index of contractility, and more recently, the characteristics of the fall of pressure have been widely used to assess relaxation. ${ }^{3}$ In our study, we aimed to investigate this problem in a homogeneous group of patients with dilated cardiomyopathy. We assessed the disturbance of activation from QRS duration, computed from the surface electrocardiogram, rather than from the usual electrocardiographic criteria of bundle branch block. We correlated the results with the characteristics of the left ventricular pressure pulse, determined noninvasively by continuous wave Doppler from the time course of functional mitral regurgitation.

\section{Patients and methods}

PATIENTS

We studied 50 patients with dilatation of the left ventricular cavity (end diastolic dimension $\geqslant 6.5 \mathrm{~cm}$ ) and reduced shortening fraction $(\leqslant 15 \%$ ), aged from 16 to 78 (mean (SD) 58 (16)) years. Five had underlying coronary artery disease and four had had an aortic valve replacement. Patients with hypertension, organic mitral valvlar disease, left ventricular aneurysm, or right bundle branch block were excluded. Twelve patients were in atrial fibrillation, and the remainder were in sinus rhythm. Functional mitral regurgitation was recorded in all.

\section{ELECTROCARDIOGRAPHY}

Standard 12 lead electrocardiograms were recorded at a paper speed of $50 \mathrm{~mm} / \mathrm{s}$ with Hewlett Packard Pagewriter XLi equipment. Computed values of $Q R S$ duration, PR interval, and $Q R S$ axis were recorded. The method of assessing QRS duration was checked against manual measurements; the mean difference between the two was $1(6) \mathrm{ms}$. 
ECHOCARDIOGRAPHY

$M$ mode echocardiograms guided from the cross sectional display were recorded with the patients in left lateral position, by a $3.0 \mathrm{MHz}$ mechanical transducer with an ATL Mark III system or $2.5 \mathrm{MHz}$ phased array transducer with a Hewlett Packard Sonos 500 system. They were recorded photographically with simultaneous electrocardiograms and phonocardiograms at a paper speed of $10 \mathrm{~cm} / \mathrm{s}$. The left ventricular $M$ mode echogram was taken at the tips of mitral leaflets, and end diastolic and systolic dimensions were taken corresponding to the start of the QRS complex and that of the first high frequency vibration of the aortic component of the second heart sound $\left(A_{2}\right)$ respectively. Shortening fraction was then derived in the usual way. Aortic ejection time was measured directly from the aortic echogram, which was also used to identify the aortic component of the second heart sound. Reversed splitting was common in patients with QRS prolongation. Mitral valve opening was taken as the point of initial mitral cusp separation. Isovolumic relaxation time was measured as the interval from $\mathrm{A}_{2}$ to mitral opening.

Doppler traces were recorded with a $2 \mathrm{MHz}$ pencil transducer and Doptek equipment, again with simultaneous electrocardiogram and phonocardiogram at a paper speed of $10 \mathrm{~cm} / \mathrm{s}$. Functional mitral regurgitation in each patient was recorded with continuous wave mode, with a 650 or $1000 \mathrm{~Hz}$ filter, from which the following measurements were made ${ }^{4}$ :

(1) RR interval.

(2) The overall duration of mitral regurgitation.

(3) Left ventricular contraction time, from the start of mitral regurgitation to the opening of the aortic valve.

(4) Aortic ejection time, from the opening of the aortic valve to its closure $\left(A_{2}\right)$.

(5) Left ventricular relaxation time, from $A_{2}$ to the end of mitral regurgitation.

We digitised the mitral regurgitation signal with time and velocity calibrations, and thus, by means of the modified Bernouilli equation, derived the time course of the pressure drop from the left ventricle to the left atrium along with its rate of change (fig 1). From these plots, we measured:

(1) Peak pressure drop from left ventricle to left atrium.

(2) Peak rate of pressure rise $(+\mathrm{dP} / \mathrm{dt})$ and fall $(-\mathrm{dP} / \mathrm{dt})$.

(3) The times to peak $+\mathrm{dP} / \mathrm{dt}$, with the start of QRS complex and mitral regurgitation as zero.

(4) The time interval from $A_{2}$ to peak negative $\mathrm{dP} / \mathrm{dt}$.

(5) The time interval from peak negative $\mathrm{dP} / \mathrm{dt}$ until mitral valve opening (isovolumic relaxation time minus $A_{2}$ to peak $-\mathrm{dP} / \mathrm{dt}$ ).

(6) Time to peak pressure-that is, when the $\mathrm{dP} / \mathrm{dt}$ curve returned to the base line.

DATA ANALYSIS

The mean value of the measurements was obtained from three successive beats and is expressed as mean (SD). The distribution of
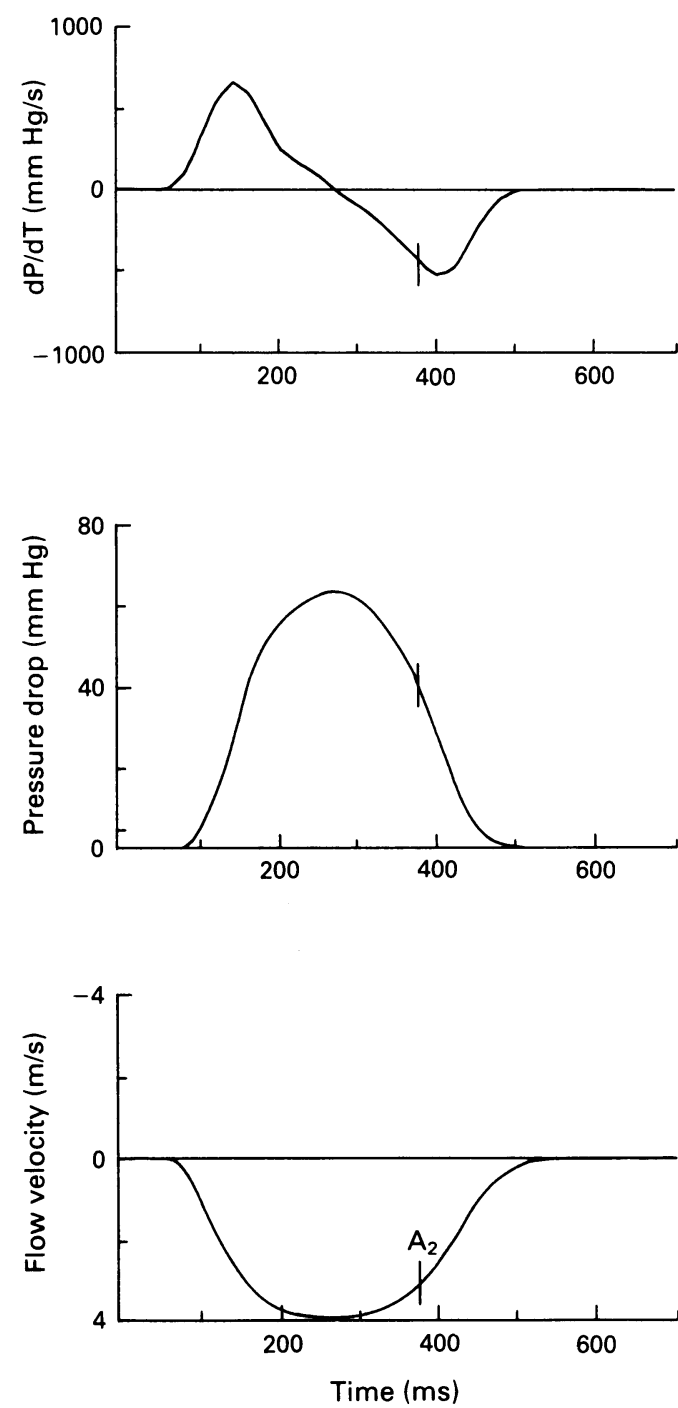

Figure 1 Doppler trace of functional mitral regurgitation (bottom trace) with calculated pressure drop (middle trace) and its first derivative (top trace). Zero time represents the start of $Q R S$ complex, $A_{2}$ represents the time of aortic closure.

values of $Q R S$ duration within the sample was analysed by a method of normal probability plot. ${ }^{5}$ Differences between mean values were assessed by Student's $t$ test and linear correlation was performed by the method of least squares. Stepwide regression and $\chi^{2}$ analyses were also performed as appropriate.

\section{Results}

Table 1 gives general electromechanical measurements.

\section{QRS DURATION}

The values of QRS duration in individual patients ranged widely, from 70 to $190 \mathrm{~ms}$ with a mean value of $110 \mathrm{~ms}$. With a normal probability plot method, ${ }^{5}$ the distribution $(r=0.984)$ did not differ significantly from normal ( $r=0.976, \alpha=0.05)$. Left axis deviaion was present in patients with normal $Q R S$ complexes, though it was commoner in patients with broad QRS complexes (fig 2). Although many of those with a QRS duration of $120 \mathrm{~ms}$ or more had features suggestive of left bundle branch block, we treated QRS duration as a 
Table 1 Electromechanical measurements

\begin{tabular}{lrll}
\hline & & \multicolumn{2}{c}{ Value } \\
Index & mean $(S D)$ \\
\hline Age (y) & 58 & $(16)$ \\
Left ventricular end diastolic dimension & & \\
$\quad$ (cm) & $6 \cdot 9(0 \cdot 9)$ \\
Shortening fraction (\%) & 11 & $(1.5)$ \\
RR interval (ms) & 725 & $(195)$ \\
PR interval (ms) & 175 & $(35)$ \\
QRS duration (ms) & 110 & $(30)$ \\
QRS axis (degrees) & 16 & $(50)$ \\
Overall duration of mitral regurgitation & & \\
(ms) & 415 & $(80)$ \\
Pre-ejection contraction time (ms) & 80 & $(30)$ \\
Aortic ejection time (ms) & 245 & $(45)$ \\
Left ventricular relaxation time (ms) & 90 & $(30)$ \\
Peak pressure difference (mm Hg) & 70 & $(30)$ \\
Q to peak pressure difference (ms) & 235 & $(45)$ \\
Q to peak + dP/dt (ms) & 115 & $(30)$ \\
Start of mitral regurgitation to peak + dP/dt & & \\
$\quad$ (ms) & 50 & $(35)$ \\
Isovolumic relaxation time (ms) & 45 & $(25)$ \\
Peak + dP/dt (mm Hg/s) & 690 & $(205)$ \\
A to peak - dP/dt (ms) & 10 & $(25)$ \\
Peak - dP/dt to mitral opening (ms) & 30 & $(25)$ \\
Peak - dP/dt (mm Hg/s) & 600 & $(220)$ \\
\hline
\end{tabular}

$+\mathrm{dP} / \mathrm{dt}$, Peak rate of pressure rise; $-\mathrm{dP} / \mathrm{dt}$, peak rate of pressure fall.

single continuous variable and did not attempt to identify electrocardiographic patterns associated with pathological $Q$ waves or with block of one or more of the fasicles of the left bundle.

\section{EFFECT OF QRS DURATION ON MITRAL REGURGITATION}

Table 2 shows the overall duration of mitral regurgitation correlated positively with QRS time $(r=0.65)$ over the whole range of values studied. The slope of the relation, $1 \cdot 7$, was significantly greater than 1 .

When the duration of mitral regurgitation was divided into contraction, aortic ejection, and relaxation times, increased QRS duration increased contraction $(r=0.51)$ and relaxation $(r=0.52)$ times. The RR interval was the only other factor affecting the duration of mitral regurgitation on stepwise regression analysis, which was performed with PR interval, QRS duration, age, and left ventricular size as additional independent variables. The effect of RR interval was mediated mainly by its exclusive influence on aortic ejection time

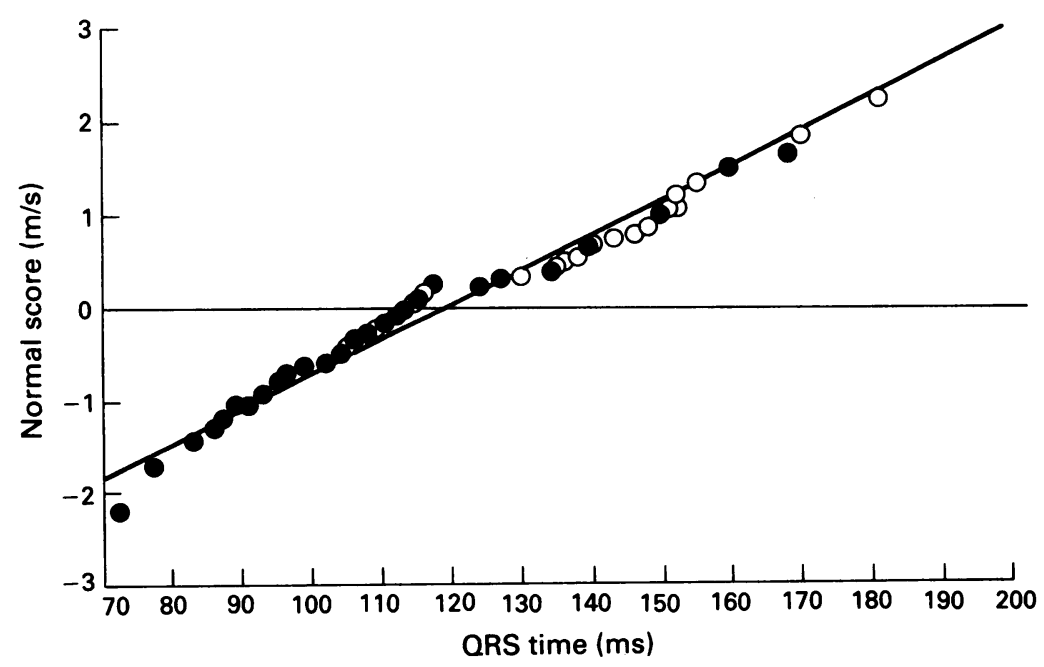

Figure 2 Normal distribution plot of $Q R S$ duration. Open symbols represent patients. Fith left axis deviation and closed symbols those without. Note that QRS duration itself is unimodally distributed throughout the sample studied although left axis deviation is commoner when $Q R S$ duration is prolonged. $(r=0 \cdot 74)$, and by a small, additional effect on relaxation time (table 3 ).

\section{EFFECT OF QRS DURATION ON DERIVED LEFT}

VENTRICULAR PRESSURE CURVE

The QRS duration correlated negatively with peak rate of rise in left ventricular pressure $(+\mathrm{dP} / \mathrm{dt})(r=-0.48)$, and positively with the time intervals from $Q$ to peak pressure $(r=0.49)$, to peak $+\mathrm{dP} / \mathrm{dt}(r=0.72)$, from the start of mitral regurgitation to peak pressure $(r=0.49)$, and to peak $+\mathrm{dP} / \mathrm{dt}$ $(r=0 \cdot 76)$. Thus when QRS duration increased, the time to peak rate of rise in pressure was prolonged and the maximal rate of the rise in pressure fell. Duration of QRS was the dominant factor influencing the times to peak $+\mathrm{dP} / \mathrm{dt} \quad\left(\mathrm{r}^{2}=52 \%\right.$ and $62 \%$, respectively). Duration of QRS, however, did not directly affect the peak rate of fall of left ventricular pressure $(-\mathrm{dP} / \mathrm{dt})$, or the isovolumic relaxation period.

\section{Discussion}

Dilatation of the left ventricular cavity with reduced ejection fraction is widely attributed to severe myocardial disease, and the impaired systolic performance explained on the basis of reduced contractility. ${ }^{2}$ Although there is no general agreement as to how this term is defined or even whether the entity exists at all, at least one approach to measuring it, $V_{\max }$, has been based on estimating rates of development of myocardial stress. ${ }^{2}$ Similarly, abnormal relaxation has been diagnosed on the basis of reduced rate of fall of pressure, measured by a time constant. ${ }^{3}$ These methods both assume that, in Wiggers' terminology, the properties of the fractional units of myocardium can be deduced directly from the properties of the ventricular pressure pulse. Wiggers' work suggested that this was only possible when activation was normal. Increased dispersion in the time of the start of activation in different regions of the ventricle may itself cause corresponding asynchrony in regional mechanical properties of the ventricle. This will reduce rates of rise and fall of ventricular pressure independently of any intrinsic myocardial disease. It was this question that we aimed to answer in our study. We chose a homogeneous group of patients with increased cavity size and reduced shortening fraction. We took advantage of recent studies indicating that when mitral regurgitation is functional and mild, the time course of the left atrium to ventricle pressure difference closely follows that of the intraventricular high fidelity pressure trace. ${ }^{6-8}$ This non-invasive approach made it possible to study a large series of patients with a wide range of QRS duration.

In the absence of rightwardly directed terminal forces, a broad QRS complex is usually described as left bundle branch block. ${ }^{9}$ This is a useful clinical term based on simple electrocardiographic pattern recognition, which identifies a group of patients with a major disturbance of activation. We used this approach in a recent study to show that when left bundle branch block was present it was associated with 
Table 2 Correlation of $Q R S$ duration ( $m s$ ) with other measurements

\begin{tabular}{|c|c|c|c|c|c|}
\hline & Slope & $\begin{array}{l}\text { (SEM) } \\
\text { slope }\end{array}$ & Intercept & $\begin{array}{l}\text { (SEM) } \\
\text { intercept }\end{array}$ & rValue \\
\hline $\begin{array}{l}\text { Overall MR duration }(\mathrm{ms}) \\
\text { Contraction time }(\mathrm{ms}) \\
\text { Aortic ejection time }(\mathrm{ms}) \\
\text { Relaxation time }(\mathrm{ms}) \\
Q \text { to peak }+\mathrm{dP} / \mathrm{dt}(\mathrm{ms}) \\
\text { Start of } \mathrm{MR} \text { to peak }+\mathrm{dP} / \mathrm{dt}(\mathrm{ms}) \\
\text { Peak }+\mathrm{dP} / \mathrm{dt}(\mathrm{mm} \mathrm{Hg} / \mathrm{s}) \\
\mathrm{Q} \text { to peak pressure }(\mathrm{ms}) \\
\text { Start of } \mathrm{MR} \text { to peak pressure }(\mathrm{ms})\end{array}$ & $\begin{array}{c}1 \cdot 7 \\
0.45 \\
0.42 \\
0.49 \\
0.61 \\
0.69 \\
-3.0 \\
0.67 \\
0.77\end{array}$ & $\begin{array}{l}(0 \cdot 28) \\
(0 \cdot 11) \\
(0 \cdot 19) \\
(0 \cdot 12) \\
(0 \cdot 11) \\
(0 \cdot 11) \\
(1 \cdot 01) \\
(0 \cdot 21) \\
(0 \cdot 24)\end{array}$ & $\begin{array}{r}230 \\
28 \\
200 \\
33 \\
48 \\
-27 \\
1040 \\
160 \\
83\end{array}$ & $\begin{array}{l}(33) \\
(12) \\
(23) \\
(14) \\
(13) \\
(13) \\
(120) \\
(25) \\
(29)\end{array}$ & $\begin{array}{r}0.65 \\
0.51 \\
0.29 \\
0.52 \\
0.72 \\
0.76 \\
-0.48 \\
0.49 \\
0.49\end{array}$ \\
\hline
\end{tabular}

MR, mitral regurgitation.

prolonged systolic activity and consequent shortening of left ventricular filling time. ${ }^{4}$ Objective analysis of QRS, however, shows not only considerable variation within patients classified as having left bundle branch block, but also in patients in whom the electrocardiographic criteria do not apply. In our study, based on a separate group of patients, we have used commercially available software to compute QRS duration, rather than the classical electrocardiographic criteria. It has shown that in patients with dilated cardiomyopathy, QRS duration is effectively unimodally distributed rather than being segregated into two groups with and without left bundle branch block. This finding obviously raises questions of definition as well as providing further evidence that the prolonged QRS duration in patients with dilated cardiomyopathy is due to an arborisation block, probably diffusely affecting the whole ventricle. ${ }^{4}$

It was possible, with echocardiography, phonocardiography and Doppler, to define a series of associations between QRS duration and the time course of the functional mitral regurgitation and thus of the pressure difference between left ventricle and atrium. Not surprisingly, the stronger correlations of QRS duration were with events early in systole-in particular the time interval from the $Q$ wave and from the start of pressure rise to peak $+\mathrm{dP} / \mathrm{dt}$ and to a lesser extent with $+\mathrm{dP} / \mathrm{dt}$ itself. The overall duration of mitral regurgitation, however, and the relaxation time ( $A_{2}$ to the end of mitral regurgitation) also correlated significantly with QRS duration, accounting for $25 \%$ or more of the total variance $\left(r^{2}=0 \cdot 27\right)$. Duration of $Q R S$ correlated only weakly with ejection time and not at all with peak negative $\mathrm{dP} / \mathrm{dt}$. We noted that mitral valve cusp separation followed the time of peak $-\mathrm{dP} / \mathrm{dt}$ by a mean value of $30 \mathrm{~ms}$, and sometimes even preceded it. We have previously shown that mitral regurgitation may continue for up to $100 \mathrm{~ms}$ after mitral cusp

Table 3 Determinants of systolic and diastolic time intervals (ms) (stepwise regression analysis)

\begin{tabular}{|c|c|c|c|c|}
\hline \multirow[b]{2}{*}{ Index } & \multirow[b]{2}{*}{ Intercept } & \multicolumn{2}{|l|}{ Slope } & \multirow{2}{*}{$\begin{array}{l}\text { Multiple } \\
\text { correlation } \\
\text { coefficient }\end{array}$} \\
\hline & & $Q R S$ & $R R$ & \\
\hline $\begin{array}{l}\text { Mitral regurgitation time } \\
\text { Filling time } \\
\text { Contraction time } \\
\text { Ejection time } \\
\text { Relaxation time }\end{array}$ & $\begin{array}{r}96 \\
-36 \\
18 \\
112 \\
-2\end{array}$ & $\begin{array}{r}1.31 \\
-1.47 \\
0.55 \\
\text { NS } \\
0.40\end{array}$ & $\begin{array}{l}0 \cdot 24 \\
0 \cdot 71 \\
\text { NS } \\
0 \cdot 17 \\
0 \cdot 06\end{array}$ & $\begin{array}{l}0.85 \\
0.93 \\
0.54 \\
0.74 \\
0.64\end{array}$ \\
\hline
\end{tabular}

NS, coefficient not significantly different from zero. separation in these patients. ${ }^{10}$ This has remained a consistent finding which we are unable to explain, but which seems to warn of complex and poorly understood factors operating at this stage in the cardiac cycle. We have thus hesitated to calculate time constants of relaxation from the Doppler derived pressure curve in our patients, particularly in the light of increasing experimental evidence suggesting that even in the normal heart, the time course of the fall in left ventricular pressure is not exponential. ${ }^{11}$

To predict the effects of activation on the pressure pulse, Wiggers developed a simple model based on algebraic summation of the contraction of the subunits. ${ }^{1}$ It suggested that increasing the period over which the summation occurs, the equivalent of prolonging the QRS duration, should not alter the time of the start of the rise in pressure, although peak rate of rise and fall would be reduced. The time intervals to peak rate of rise and peak pressure would be prolonged as would be the overall duration of the pressure pulse, each by the same amount as the increase in QRS duration, although peak pressure itself is unlikely to be changed. Our findings thus agree closely with these predictions especially with respect to early systole and the overall duration of mitral regurgitation. The main points of difference were the lack of correlation of $-\mathrm{dP} / \mathrm{dt}$ with QRS duration, although relaxation time itself correlated, and also that the extent of the prolongation of mitral regurgitation was $70 \%$ greater than that of the QRS complex itself. In general, therefore, observation agrees with prediction enough to suggest that the electromechanical correlations we describe could be explained largely on the basis of the theory of fractionate contractions.

Our results may have some practical significance especially when it comes to assessing myocardial function. Peak $\mathrm{dP} / \mathrm{dt}$, or peak $\mathrm{dP} / \mathrm{dt}$ standardised to instantaneous pressure, are still recommended as being of value in assessing myocardial systolic performance and described as contractility. ${ }^{1213}$ Clearly such estimates depend critically on the pattern of ventricular activation. This was not allowed for in the original studies, an omission that may contribute to the poor performance of such isovolumic indices in identifying patients with reduced systolic function. Also, acute change in $\mathrm{dP} / \mathrm{dt}, \mathrm{dP} / \mathrm{dt}$ standardised to instantaneous pressure, or time to peak $\mathrm{dP} / \mathrm{dt}$ are often used to assess the effect of drugs. Should the drug also prolong QRS duration, then effects on the 
pressure curve would be expected as the direct consequence of the electrophysiological change and need not be attributed to depressed function of individual myocardial units. ${ }^{14} 15$

In summary, therefore, our resuts show the potential value of taking QRS duration into account in assessing both systolic and diastolic myocardial function, in patients with dilated cardiomyopathy. The QRS duration is routinely computed and printed out by modern electrocardiographs; such values are little used clinically, but are clearly significant once their consequences in terms of the severity of the haemodynamic disturbance in individual patients are considered. Further, if QRS duration could be reduced, possibly by an appropriate pacing technique, the equivalent of a positive inotropic effect on rates of both contraction and relaxation could be achieved without the complications of tachyphylaxis, increasing myocardial oxygen requirements, or a proarrhythmic effect. We feel that these electromechanical interactions are worthy of study.

HBX is supported by The Brompton Hospital Special Cardiac Fund; SJDB is supported by a British Heart Foundation Junior Research Fellowship.

1 Wiggers CJ. Are ventricular conduction changes important in the dynamics of ventricular contraction? Am J Physiol in the dynamics

2 Ross J Jr. Cardiac function and myocardial contractility: a perspective. J Am Coll Cardiol 1983;1:52-63.
3 Weisfeldt ML, Weiss JL, Frederisken JT, Yin FCP. Quantification of incomplete left ventricular relaxation: relationship to the time constant for isovolumic pressure fall. Eur J Heart 1980;1(suppl A):119-29.

$4 \mathrm{Xiao}$ HB, Lee CH, Gibson DG. Effect of left bundle branch block on diastolic function in dilated cardiomyopathy. $\mathrm{Br}$ Heart J 1991;66:443-7.

5 Cruze E, Weldon J. Normal probability plots. In: Cruze E, Hartzell B, eds. Minitab reference manual release 8 . Pennsylvania: Minitab Inc, 1989:4.8.

6 Bargiggia GS, Bertucci C, Recusani F, Raisaro A, Servi SD, Valdes-Cruz LM, et al. A new method for estimating left ventricular $\mathrm{dP} / \mathrm{dt}$ by continuous wave Doppler echocardiography. Circulation 1989;80:1287-92.

7 Neumann A, Korcarz C, Lang RM, Sareli P, Marcus R, Borrow KM. Comparison of Doppler vs catheterization derived $\mathrm{dP} / \mathrm{dt}$ in dilated cardiomyopathy [abstract]. Circulation 1989;80(suppl II):170.

8 Chen C, Rodriguez L, Guerero JL, Marshall S, Levine RA, Weyman AE, Thomas JD. Noninvasive estimation of the instantaneous first derivative of left ventricular pressure using continuous-wave Doppler echocardiography. using continuous-wave Dopp

9 Rowlands DJ. Left bundle branch block. In: Julian DG, et al. Diseases of the Heart. London: Bailliere Tindall, 1989: $157-9$.

$10 \mathrm{Ng} \mathrm{KSK}$, Gibson DG. Impairment of diastolic function by shortened filling period in severe left ventricular disease. Br Heart J 1989;62:246-52.

11 Nikolic S, Yellin EL, Tamura K, Tamura T, Frater RWM Effect of early diastolic loading on myocardial relaxation in the intact canine left ventricle. Circ Res 1990;66:1217-26.

12 Grossman W. Evaluation of systolic and diastolic function of myocardium. In: Grossman, ed. Cardiac Catheterization and Angiography, 4th ed. Philadelphia: Lea and Febiger, 1991:319-39.

13 Milnor W. Myocardial contractility. In: Milnor W, ed Cardiovascular physiology. Oxford: Oxford University Press, 1990:100-2, 125-7.

14 Legrand V, Vandormael M, Collignon P, Kulbertus HE Hemodynamic effects of a new antiarrhythmic agent, Flecainide (R-818), in coronary heart disease. Am Cardiol 1983;51:423-6.

15 Josephson MA, Ikeda N, Singh BN. Effect of flecainide on ventricular function: clinical and experimental correlations. Am J Cardiol 1984;53:95-100. 